\title{
Cooperative Architecture for Cellular-short-range Combined Mesh Networks
}

\author{
(Invited Paper) \\ Tao Chen \\ VTT Technical Research Centre of Finland \\ P.O. Box 1100, FI-90571 \\ Oulu, Finland \\ tao.chen@vtt.fi \\ Marcos D. Katz \\ VTT Technical Research Centre of Finland \\ P.O. Box 1100, FI-90571 \\ Oulu, Finland \\ marcos.katz@vtt.fi
}

\begin{abstract}
Due to the nature of wireless communications, a single radio access technology (RAT) hardly satisfies all communication needs. RATs are roughly divided into two types: cellular and short-range. It becomes increasing popular to equip a mobile device with both types of air interfaces. By seamlessly integrating different types of air interfaces into a single network, a new mobile communication model emerges. Benefit from the mesh structure interweaved by cellular and short-range links, the new model is able to enjoy reliability, flexibility, coverage extension and enhanced service. Although several architectures have been proposed based on this model, a practical control solution for seamless integration of cellular and short-range systems is still absent. We propose in this paper a novel cooperative architecture with a control plane backed by cellular systems. A dedicated control unit is developed to tightly control the user cooperation of mobile devices using their short-range links. In addition to the architecture design, we propose two services based on the proposed architecture. It shows the potential of the architecture to carry new services, especially, multimedia services.
\end{abstract}

\section{Categories and Subject Descriptors}

C.2.1 [Computer-Communication Networks]: Network Architecture and Design

\section{General Terms}

Design

\section{INTRODUCTION}

Mobile communications over last two decades have experienced an explosive growth. Up to date there are more than 3.4 billion mobile phones around world. Accompanying with the rapid increase of numbers comes the function transform of mobile phones. Nowadays a mobile phone is no long a

Permission to make digital or hard copies of all or part of this work for personal or classroom use is granted without fee provided that copies are not made or distributed for profit or commercial advantage and that copies bear this notice and the full citation on the first page. To copy otherwise, or republish, to post on servers or to redistribute to lists, requires prior specific permission and/or a fee.

Mobimedia'09, September 7-9, 2009, London, UK

Copyright 2009 ICST 978-963-9799-62-2/00/0004 ...\$5.00. pure voice terminal but becomes a general-purpose personal mobile computer, in which data communications become an integral part. To facilitate data communications, it becomes a trend to install multiple air interfaces on a single mobile device, e.g., cellular, Bluetooth or WiFi. It gives a mobile device means to access different type of networks, which can complement each other according to their own characteristics. Evidence shows that cooperation of multiple air interfaces of a mobile device is able to provide significant service gain [4]. Cooperation through short-range air interfaces of mobile devices actually creates a new paradigm for cellular communications [4]. However, the architecture of current cellular networks has less consideration for this paradigm. To fully release the power of cellular-short-range cooperation, which has potential to significantly improve the resource utilization of cellular networks, enhancement to current point to multi-points (PMP) architecture is demanded.

In the past, cellular and short-range networks are normally combined only for capacity enhancement and coverage extension (see [1],[6], [11],[9],[8] and references therein). An ad-hoc extension for second-generation GSM was suggested in [1], where mobile stations (MS) take part in relaying using their Mobile Ad Hoc Network (MANET) interfaces to improve the coverage of the GSM system. Wei et al. [11] integrated WWAN and WLAN into a two-hop-relay architecture for the system capacity improvement of WWAN systems and coverage extension of WLAN terminals. In [12], a cellular system is integrated with ad hoc relays to achieve load-balancing in cellular networks. Luo et al. [8] used ad hoc networks to improve the capacity of cellular networks. Moreover, relay-based systems have been intensively studied by the working group 4 of the wireless world research forum (WWRF) [9]. As we can see, most proposed approaches simply aim to find new possible ways to reach end users from the cellular network. The opportunity obtained from the user cooperation in short-range networks is seldom considered in the architecture design.

To fill this gap, an architecture enabling user cooperation cross cellular and short-range links was proposed by Fitzek et al. [5]. Named as cellular controlled peep to peer communications, the essential idea of this cooperative architecture is to establish a wireless grid weaved by cellular and shortrange links of mobile devices, allowing different types/levels of resources being shared in the grid. Service enhancement instead of coverage extension becomes the primary focus of 


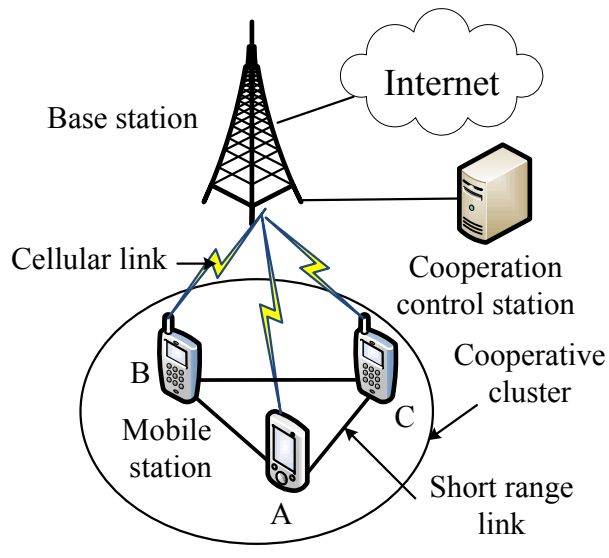

Figure 1: Cooperative Architecture.

the architecture. The use of this architecture to achieve energy saving, improve the quality of DVB-H streaming, and perform cooperative tasks was demonstrated in [2],[4].

The architecture proposed in [5] may give a new life to conventional cellular networks. It turns conventional cellular networks into a mesh network where radio and network resources can be flexibly provisioned according to needs. To release the power of the architecture, a control plane capable of seamlessly integrating the cellular and short-range links becomes necessary. Otherwise, the benefits brought by the architecture may be compromised. To our best knowledge, a practical control solution is still missing. It is the objective of this paper to propose a control solution for the architecture and give birth to a new cooperative architecture.

The contributions of the paper are following:

- A control architecture is proposed, where cellular links are used as control channels and a dedicated control unit, namely the cooperation control station (CCS), is designed to fully coordinate cooperation in proposed architecture.

- The idea of cellular link aggregation is proposed based on the designed control architecture. Multiple cellular links are able to aggregate to serve one MS through the cooperation of other MSs.

- Two interesting cooperation services are introduced based on the proposed architecture.

The remainder of the paper is organized as follows. Section 2 explains the architecture, which includes the detailed description of the CCS. Section 3 gives the control procedures of two cooperation services. We then draw the conclusion and give future work in Section 4 .

\section{ARCHITECTURE}

As shown in Fig. 1, the cooperative architecture comprises cellular base stations (BS), multi-air-interface MS, and a CCS. A BS offers to its MSs packet-based voice, video and data services. We focus on multimedia services over Internet in this paper. MSs access services mainly through cellular links. This is a typical case in current cellular networks. MSs are assumed to have one or several short-range air interfaces, e.g. Bluetooth or WiFi. Indeed, mobile phones with multiple air interfaces become increasing popular. It is reasonable to assume that the majority of future mobile devices will be equipped with multiple air interfaces. The CCS is proposed as the key to facilitate mobile cooperation. With the aid of the CCS, a reliable mesh topology is established and maintained on a demand basis among BSs and MSs through their cellular and short-range links. On top of this topology run conventional, improved, and new services. In this architecture, the edge of wireless access networks disappears. MSs are not any more the terminals of services, but the flexible extension of service infrastructure.

The following example shows a typical cooperation process occurring in the architecture. As shown in Fig. 1, the MS A, $B, C$ register on the CCS through their cellular links. With the assistance of the CCS, the MS $A$ knows the MS $B$ and $C$ are its one-hop neighbors who are willing to share their resources with the MS $A$. At a moment, the user of the MS $A$ wants to watch a high-quality online video. However, the available bandwidth of this mobile may not always guarantee the bandwidth required by this video. By checking the cooperation opportunity from the CCS, the mobile knows additional bandwidth can be borrowed from the MS $B$ and $C$ through their short-range links. The MS $A$ then negotiates with the MS $B$ and $C$ through the CCS and starts the cooperation session from the CCS.

As we can see from the example, several problems have to be addressed before making a seamless cooperation possible. First of all, a signaling mechanism is required to setup and control the cooperation. We propose the use of cellular links as the control channels and the CCS as the point to perform the control task. The CCS not only provides signaling for short-range cooperation of MSs, but also acts as a gateway to make the cooperation transparent to the transport layer of the network stack. To enable the signaling in this architecture, the modification of the protocol stack in the CCS and MSs is required. We propose a 2.5 layer in the CCS and MSs and use it to tunnel the transport layer services. The aim is to minimize the modification to the current protocol stack. To facilitate the cooperation, we introduce the cooperative cluster concept as well. A cooperative cluster is a set of mobile devices grouped on demand for a cooperation session. We describe the cooperative cluster, CCS, and protocol stack as follows.

\subsection{CCS}

The CCS is responsible for cooperation management in the proposed architecture. The motivation to introduce the CCS is to fully release the power of cooperation in cellular-shortrange networks. In most proposed approaches [4], the MS is responsible for the organization of cooperation. It is not an efficient solution since a MS has limited view on resource allocation of network. Additional management tasks may consume significant amount of battery energy from MSs. Moreover, the always-on cellular links of MSs, which are the best for the control purpose, are not explored at all to facilitate the cooperation. In addition to the control problem, 


\section{Cooperation control station}

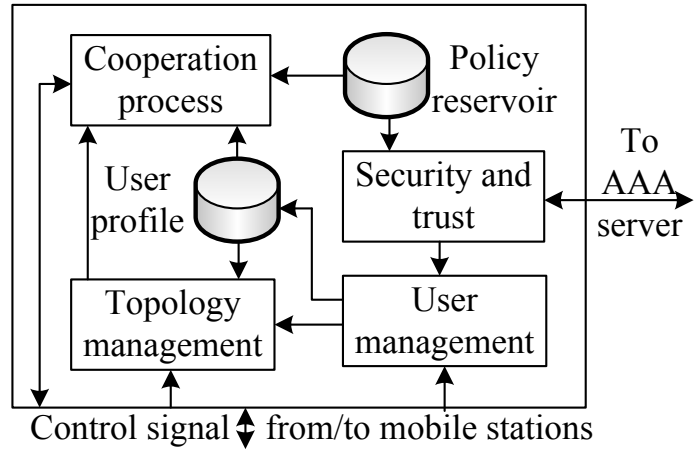

Figure 2: Cooperation control station.

the security problem is rarely touched by existing solutions. In practice not every MS is willing to join any kind of cooperation. The use of the CCS has potential to solve all those problems.

The CCS is composed of a control and data plane. The main functions of the control plane include: user management, topology management, cooperation process, policy enforcement, and security and trust. In the data plane, the CCS acts as a gateway for transparent transport layer services between MSs and service servers. The building blocks of the CCS are shown in Fig. 2.

\subsubsection{Control plane}

The user management module takes the responsibility of user registration and de-registration. Note that the user and MS are different concepts in this paper. Similar in $2 \mathrm{G}$ and $3 \mathrm{G}$ networks where a user is represented by a subscriber identity module (SIM) card and can be mounted on different mobile phones, we use the same idea here to allow users define their device-independent cooperation policies. This separation of concern simplifies the management task and makes it possible to run cooperation services by operators.

Through the security and trust module, the user management module is able to authenticate the user. The security and trust module has connection with the authentication, authorization, and accounting (AAA) server of the cellular network. The authentication of the user is performed remotely in the AAA server. In addition, the security and trust model retrieves security policies from the policy reservoir, computes the constraints of users in a cooperation session, and instructs the cooperation process unit to solve security conflicts in cooperative clusters.

After a successful registration, a user is associated with stored cooperation police. The cooperation policies define the constraints a user will obey when cooperating with others. The user management module also collects the capabilities of the MS, which include the parameters of air interfaces and the status of the MS, e.g. the battery level and its location in the cellular network. It stores the collected information into the user profile database, polls and updates the status of the MS periodically until it de-registers from the CCS.

The topology management module is responsible for obtaining the potential topology of MSs formed by their shortrange links and providing the cooperation process unit the topology information to create and maintain cooperation sessions. The potential topology means the complete connectivity map generated by the short-range air interfaces of the MSs which are allowed by their users for cooperation. It indicates possible cooperative clusters upon which could be built. Due to the mobility of MSs or other reasons, the potential topology may experience dynamic changes, and in turn affect the setup of cooperation. It thus requires mechanisms to timely update the potential topology. To achieve this, the topology management module uses neighboring discovery and link monitoring as proactive approaches to discover and maintain the topology.

By knowing the approximate location and air interface information of MSs during the registration phase, the topology management module instructs MSs to perform neighboring discovery intelligently. Indeed, having a top view of MSs in the CCS allows a more efficient neighboring discovery process among MSs. The neighboring discovery process is performed regularly in each MS. Once detecting new neighbors, a MS starts the link monitoring process to update the connectivity with its neighbors periodically. The topology management module instructs MSs the frequency to perform their link monitoring according to the cooperation needs. In addition to proactive topology monitoring approaches, the topology management module receives de-registration notifications from the user management module and updates the topology accordingly.

The cooperation process unit performs the control task in a cooperation session. Once receiving a cooperation request from a MS, it first checks from the potential topology the possible cooperation partners. It then obtains the cooperation policies of those partners from the policy reservoir, and their air interface configuration and status from the user profile database, calculating the possible cooperative cluster setups. The cooperation policies are pre-defined rules representing the preference of users to share their MSs resources. Those policies are agreements setup between users and operators, defining the permissions that the CCS can use to automatically setup cooperation sessions. Policies are represented by a semantic language that allows computation. There are several policy language candidates to fulfill this need [3],[10],[7].

The algorithm in the cooperation process unit determines the configuration of the cooperation, which includes the shortrange interfaces to be used, the cooperation link, and the setup of each MS in the cluster. Since an associated MS in a cluster may acts as a relay node forwarding packets between different air interfaces, it is important to build a correct packet-forwarding mechanism. To achieve this, the modification of the network protocol stack in MSs is required. We will explain the detail of the protocol stack in the following section.

The cooperation process unit instructs the MSs to setup a 


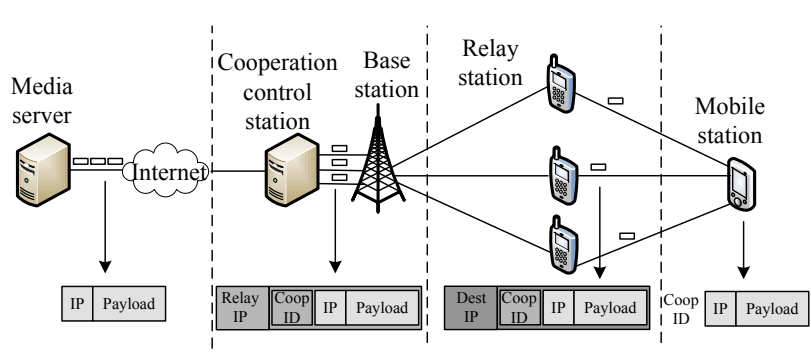

Figure 3: A cooperation procedure.

cooperative cluster once it obtains the configuration from its algorithm. Note that the algorithm may conclude that there is no suitable configuration for the requested cooperation. Moreover, the configuration may change during the lifetime of the session triggered by some events, for instance, an associated MS leaving the reach range of the cluster, or a new MS joining the cluster. The cooperation process unit needs to monitor the topology and update the cluster configuration accordingly.

\subsubsection{Data plane}

One important service the CCS can offer is the bandwidth sharing service among MSs. As partially illustrated in Fig. 3, the service allows the transport layer stream being split at the CCS, relayed by several MSs through their shortrange links, and finally aggregated at the destination MS. Although Fig. 3 only shows a unicast case, the multicast and network coding based applications can be supported by this service. The bandwidth sharing service is enabled by the data plane of the CCS.

The data plane of the CCS acts as a gateway to provide transparent transport layer services between MSs and service servers. It provides two modules: transport layer tunneling, and multi-stream scheduling. The transport layer tunneling module splits a single data stream from the service server to multiple streams according to the cooperative cluster configuration and load balancing requirement. The split multi-streams are tunneled into a 2.5 layer of the protocol stack and resembled at the destination MS. The behaviors of the data plane are controlled by the cooperation process unit of the control plane and is able to adapt to the dynamic topology changes. To avoid packet loss due to unexpected topology changes, a buffer is offered per cooperation session. The multi-stream scheduling module schedules the load to multi-streams according to the channel quality information of cellular and short-range links.

\subsection{Protocol stack}

To support the split and aggregation of transport streams over multi-hop links, it requires the nodes along the path between the CCS and the destination MS acting as a router. Such a router should be able to dynamically change their configuration according to the need of a cooperative cluster. The reconfiguration is time-critical. Otherwise the bandwidth sharing service of the CCS will fail. Conventional routing schemes in wired and wireless ad hoc network hardly meet this requirement. Inspired from the multi-protocol label switching (MPLS) concept, we introduce a 2.5 layer ap-

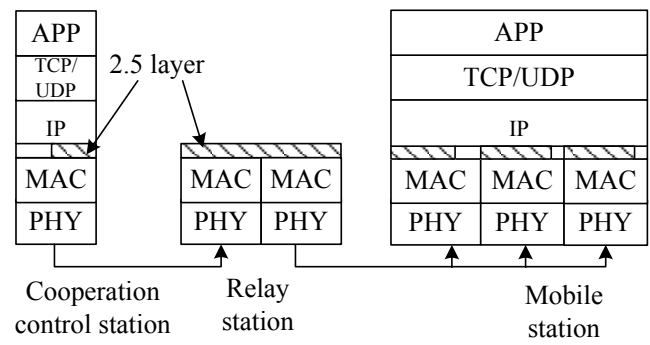

Figure 4: Protocol stack of proposed architecture.

proach in the proposed architecture. In such an approach, an additional sub-layer is embedded in the IP layer of the CCS and MSs, responsible for routing across cooperative clusters. The objective of this design is to maximize the compatibility by keeping other layers untouched. The modified protocol stack is shown in Fig. 4.

As shown in Fig. 3, the CCS assigns each cooperation session a cooperation ID, which is used in the 2.5 layer for the routing purpose. When the data plane of the CCS receives an IP packet destined to a cooperative cluster, it checks from the scheduling rule which MS in the cluster will relay the packet. Once determining the relay MS, the data plane of the CCS encapsulates the whole packet into a new IP packet with the IP address of the relay MS as the destination address. The cooperation ID and other control information are embedded at the beginning of the payload of the new IP packet, followed by the original IP packet. The source address of the new generated IP packet is set using the IP address of the CCS. By this means the relay MS is able to identify it as a cooperative packet. The control plane of the CCS configures the MSs in each cooperation cluster properly before and during the cooperative session. The relay MS therefore knows from which air interfaces the packet will be forwarded. The packet is finally delivered to the destination MS. From the cooperation ID in the packet, it knows how to deliver the packet to the correct stream in the IP layer. If the transport layer service needs acknowledgement, acknowledgement packets are sent directly from the destination MS through its cellular link.

If no cooperation is requested by the MS, the 2.5 layer simply bypasses the packet without any re-encapsulation process. It is shown in Fig. 4 that the blank part of the 2.5 layer bypasses the packet, while the shadow part the 2.5 layer involves in the cooperation process. The CCS is responsible for configuring the 2.5 layer of a MS to switch on/off the cooperation.

\subsection{Cooperative cluster}

The cooperation is managed by clusters. A cooperative cluster is formed by the CCS according to the request of one or several MSs. One MS can request the setup of a unicast cooperative cluster where other MSs in the cluster are relay nodes. Multiple MSs can initialize a multicast cooperative cluster where the members of cluster act as both relays and receivers. A cluster can be terminated either by the requested MS, or by the CCS when a raising policy conflict requests a termination. For instance, the battery levels of 
all relay MSs are lower than their thresholds.

The CCS assigns a unique cooperation ID to each cooperative cluster. A MS may participate in multiple clusters, each of them having a unique cooperation ID. For instance, a MS may setup a cluster where it receives resource from others, and meanwhile it offers resource to others in another cluster. The cooperation ID is used in the 2.5 layer to route packets cross multiple air interfaces of MSs. Section 2.2 explains how the cooperation ID is embedded in the IP packet for this purpose.

The behaviors of a cooperative cluster are fully controlled by the CCS. Through the cellular link, the CCS is able to configure the 2.5 layer of each MS in a cluster. Therefore packets are routed correctly cross multiple air interfaces of MSs. Using neighboring discovery and link monitoring functions, the CCS is able to obtain the changes of short-range links, and then adapt clusters to those changes through reconfiguration.

\section{CONTROL PROCEDURE}

In this section we introduce the control procedures of two different applications which can significantly benefit from the proposed cooperative architecture. One is the direct peer to peer sharing in which two neighboring MSs use their short-range link to exchange files and messages. Energy is saved by using the short-range link instead of the cellular link. The other is the link aggregation through bandwidth sharing. We have briefly introduced the bandwidth sharing concept in the aforementioned section. The detailed procedure is provided in this section.

\subsection{Peer to peer sharing}

One may wonder that since two MSs can be reached by a short-range link why the proposed architecture is needed for their peer to peer sharing. There are two reasons justifying the use of the cooperative architecture. First, The proposed architecture is able to sense their short-range connectivity and setup the short-range link on demand. The user intervention to setup the connection is minimized. The second reason is that when the short-range link is broken, the proposed architecture is able to switch to the cellular links so that the connection keeps alive.

The control procedure for a peer to peer sharing is shown in Fig. 5 and described as follows:

1. Two MSs, denoted by $A, B$, register on the CCS.

2. The MS $A$ initializes a peer to peer connection with $B$ through the CCS.

3. Through the neighboring discovery, the CCS detects the MS $A$ and $B$ are one-hop neighbors connected by a short-range link.

4. The CCS configures the 2.5 layer of the MS $A$ and $B$ to route packets of the peer to peer connection directly through the short-range link.

5. The peer to peer connection starts.

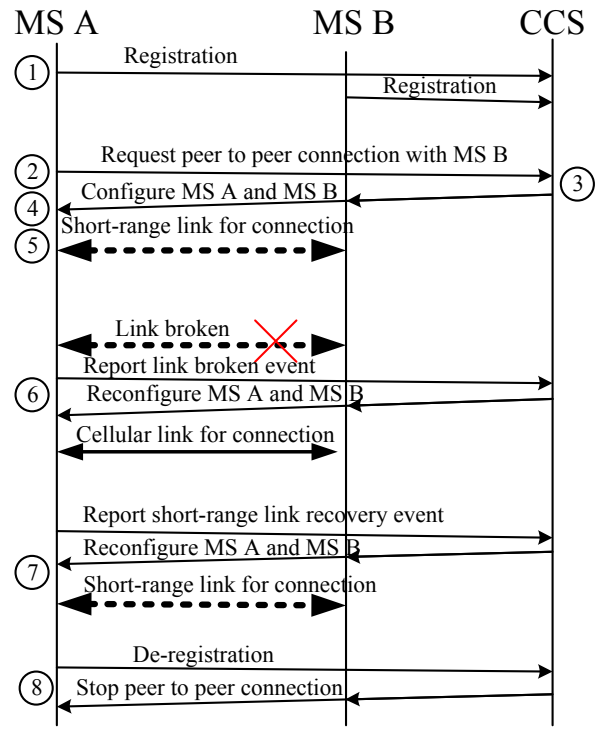

Figure 5: Control procedure for peer to peer sharing.

6. The CCS monitors the short-range link during the peer to peer session. Once the link broken event is detected, it reconfigures the 2.5 layer of both MSs to redirect peer to peer connection through the cellular links.

7. When the CCS detects that the short-range link is recovered, the peer to peer connection is shifted back to the short-range link.

8. A MS requests to stop the peer to peer sharing. The CCS cleans the cooperation configure for two MSs.

\subsection{Link aggregation by bandwidth sharing}

In a cellular network, normally a user signs a service level agreement (SLA) with the operator. The SLA defines maximum bandwidth or total traffic the user can use during a given period. The link aggregation through bandwidth sharing in a cellular network becomes reasonable since it gives users an opportunity to re-allocate resources among trusted parties. Moreover, the bandwidth sharing provides a solution to improve the connection when a MS has poor link quality to the cellular network. The control procedure of a bandwidth sharing service is shown in Fig. 6 and explained in the following:

1. MSs, denoted by $A, B$, and $C$ register on the CCS.

2. The MS $A$ requests a bandwidth sharing service on the CCS.

3. After a searching, the CCS finds the MS $B$ and $C$ can offer their bandwidth to the MS $A$.

4. The CCS configures the 2.5 layer of MS $A, B$ and $C$ to forward and receive packets correctly. The cluster is formed. 


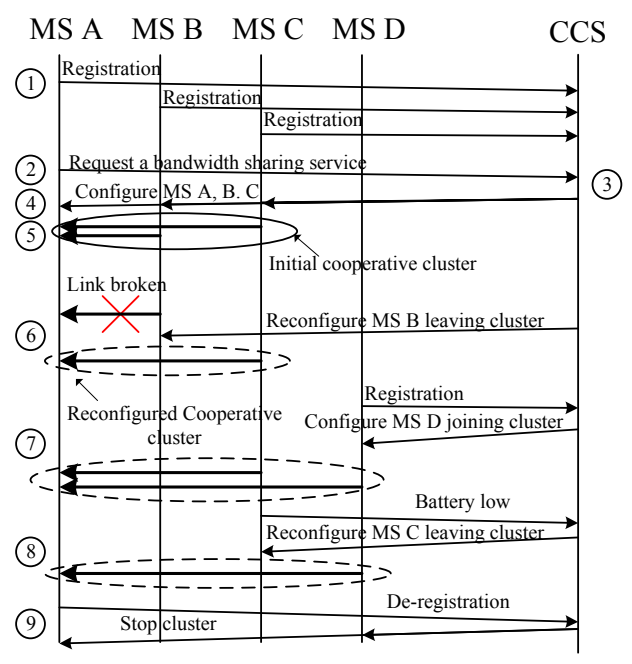

Figure 6: Control procedure for bandwidth sharing.

5. The MS A starts network applications using link aggregation.

6. The CCS monitors the short-range links during the cooperative session. It reconfigures the cluster once detecting link changes. In Fig. 6 , the link between the MS $A$ and $B$ is broken.

7. The CCS detects a new MS $D$ can share its bandwidth to the MS $A$. It configures the MS $D$ to join the cluster.

8. The battery level of the MS $C$ drops below a threshold defined in a policy of the MS $C$. The CCS releases the MS $C$ from the cluster.

9. The MS $A$ requests a de-registration from the CCS. The CCS terminates the cluster.

\section{CONCLUSION}

The introduction of the cooperative architecture actually turns the conventional PMP cellular architecture into a mesh structure where MSs are able to interweave their short-range links with cellular links. It provides a considerable solution for heterogeneous network integration with a general goal to increase the efficiency of resource utilization. The use of the dedicated CCS provides a chance to manage different resources at network wide, thus increasing flexibility and reliability of resource sharing. Moreover, the top view of the CCS on network resources enables better solutions to optimize the resource usage. In addition to achieving energy saving and enabling new services, the proposed architecture is able to coordinate the spectrum use in neighboring cooperation clusters. Indeed, the common control channel problem has been a challenge in dynamic spectrum access (DSA) networks. In the proposed architecture, the cellular link actually acts as a common control channel and the CCS becomes the spectrum coordinator. In future work we will apply the architecture to DSA scenarios.

In addition to the benefits, the proposed architecture may bring several problems to current cellular networks. The mobility of MSs may cause stability problems in cooperation and thus affect the QoS of network applications. The multi-streams solution in short-range networks may introduce additional latency and jitter, which in turn impact the transport layer services. Moreover, the proposed architecture requires additional control overhead over cellular links. It costs on one hand the cellular link bandwidth, and on the other hand the energy of MSs. We will investigate those problems in the future work.

\section{ACKNOWLEDGMENTS}

The work was supported by the CR-MAP project, which is fully funded by VTT Technical Center of Finland.

\section{REFERENCES}

[1] G. Aggelou and R. Tafazolli. On the Relaying Capability of Next-generation GSM Cellular Networks. IEEE Personal Communications, 8(1):40-47, 2001.

[2] F. Albiero, M. Katz, and F. Fitzek. Energy-Efficient Cooperative Techniques for Multimedia Services over Future Wireless Networks. IEEE ICC'08, Beijing, China, May 2008.

[3] DARPA XG Working Group. XG Policy Language Framework. Request for Comments. Version 1.0, Prepared by BBN Technologies, Cambridge MA, USA, April 2004.

[4] F. Fitzek and M. Katz. Cognitive Wireless Networks Concepts, Methodologies and Visions: Inspiring the Age of Enlightenment of Wireless Communications. Springer, 2007.

[5] F. Fitzek, M. Katz, and Q. Zhang. Cellular Controlled Short-range Communication for Cooperative P2P Networking. Wireless Personal Communications, 48(1):141-155, 2009.

[6] H. Hsieh and R. Sivakumar. On Using the Ad-hoc Network Model in Cellular Packet Data Networks. ACM MOBIHOC'02, Lausanne, Switzerland, June 2002.

[7] IEEE 1900.5 Working Group. IEEE SCC41. http://grouper.ieee.org/groups/scc41/5/index.htm (2009).

[8] H. Luo, R. Ramjee, P. Sinha, L. Li, and S. Lu. UCAN: a Unified Cellular and Ad-hoc Network Architecture. ACM MOBICOM'03, California, USA, September 2003.

[9] R. Pabst, B. Walke, D. Schultz, et al. Relay-based Deployment Concepts for Wireless and Mobile Broadband Radio. IEEE Communications Magazine, 42(9):80-89, 2004.

[10] Software Defined Radio Forum. Metalanguage for Mobility Work Group. http://www.sdrforum.org (2009).

[11] H. Wei and R. Gitlin. Two-hop-relay Architecture for Next-generation WWAN/WLAN Integration. IEEE Wireless Communications, 11(2):24-30, 2004.

[12] H. Wu, C. Qiao, S. De, and O. Tonguz. Integrated Cellular and Ad Hoc Relaying Systems: iCAR. IEEE Journal on Selected Areas in Communications, 19(10):2105-2115, 2001. 